JOURNAL OF INTEGRAL EQUATIONS

AND APPLICATIONS

Volume 10, Number 4, Winter 1998

\title{
NUMERICAL CONFORMAL MAPPING FOR EXTERIOR REGIONS VIA THE KERZMAN-STEIN KERNEL
}

\author{
A.H.M. MURID, M.Z. NASHED AND M.R.M. RAZALI
}

Dedicated to Philip M. Anselone, in appreciation of a long friendship and lasting contributions

ABSTRACT. A simple numerical method is described for computing a conformal map from a domain exterior to a smooth Jordan curve in the complex plane onto the exterior of the unit disk. The numerical method is based on a boundary integral equation similar to the Kerzman-Stein integral equation for interior mappings. Typical examples show that numerical results of high accuracy can be obtained provided that the boundaries are smooth.

1. Introduction. Let $\Gamma$ be a Jordan curve, $\Omega^{+}$and $\Omega^{-}$be the interior and exterior of $\Gamma$, respectively. It is well known, see, e.g., $[\mathbf{7}, \mathrm{p}$. $374]$, that there exists a unique function $\tilde{R}$, called the exterior mapping function of $\Omega$, which maps $\Omega^{-}$onto the exterior of the unit disk such that $\tilde{R}(\infty):=\lim _{z \rightarrow \infty} \tilde{R}(z)=\infty$ and the Laurent series of $\tilde{R}$ at $\infty$ has the form

$$
\tilde{R}(z)=\gamma^{-1} z+a_{0}+a_{1} z^{-1}+\cdots, \quad \gamma>0, z \in \Omega^{-} .
$$

The positive constant $\gamma$ is called the capacity of $\Gamma$, or the transfinite diameter of $\Omega^{+}$.

Since $\Gamma$ is a Jordan curve, $\tilde{R}$ can be extended to a homeomorphism of the closure $\Omega^{-} \cup \Gamma$ to the closure $|w| \geq 1$. Thus as $z(t)$ traverses along $\Gamma$, the image point $\tilde{R}(z(t))$ describes the unit circle. Hence

$$
\tilde{R}(z(t))=e^{i \theta(t)}, \quad 0 \leq t \leq \beta,
$$

1991 AMS Mathematics Subject Classification. Primary 30C30, Secondary 45B05.

Key words and phrases. Conformal mapping, exterior mapping function, integral equation, Kerzman-Stein kernel, Nyström method.

Copyright (C) 1998 Rocky Mountain Mathematics Consortium 
or

$$
\theta(t)=\arg \tilde{R}(z(t)), \quad 0 \leq t \leq \beta
$$

The function $\theta$ is called an exterior boundary correspondence function.

The function $\tilde{R}$ is expressible in terms of $\theta$ by the formula [7, p. 381]

$$
\tilde{R}(z)=\left[\frac{z}{2 \pi i} \int_{0}^{\beta} \frac{e^{-i \theta(t)}}{z-z(t)} \frac{z^{\prime}(t)}{z(t)} d t\right]^{-1}, \quad z \in \Omega^{-}, z(t) \in \Gamma, 0 \leq t \leq \beta .
$$

If $\theta^{\prime}$ is also known, then the inverse $\tilde{R}^{-1}$ is expressible in terms of $\theta$ and $\theta^{\prime}$ by means of

$$
\tilde{R}^{-1}(w)=\left[\frac{1}{2 \pi} \int_{0}^{\beta} \frac{1}{z(t)} \frac{1}{1-w^{-1} e^{i \theta(t)}} \theta^{\prime}(t) d t\right]^{-1}, \quad w \in\{w:|w|>1\} .
$$

For sufficiently regular boundaries, the functions $\theta$ and $\theta^{\prime}$ are known to satisfy certain integral equations, some of which are given below.

Symm's exterior equation $[\mathbf{1 6} ; \mathbf{7}$, p. 385].

(1.6) $\frac{1}{2 \pi} \int_{0}^{\beta} \log \left|1-\frac{z(t)}{z(s)}\right| \theta^{\prime}(t) d t=\log \gamma-\log |z(s)|, \quad 0 \leq s \leq \beta$.

Banin's integral equation [7, p. 397].

$$
\theta^{\prime}(s)-\int_{0}^{\beta} v(s, t) \theta^{\prime}(t) d t=0, \quad 0 \leq s \leq \beta .
$$

Equation of Kantorovich-Krylov [7, p. 397].

$$
\theta(s)+\int_{0}^{\beta} v(t, s) \theta(t) d t=2 \arg [z(s)-z(0)], \quad 0 \leq s \leq \beta .
$$


The kernel $v$ appearing in (1.7) and (1.8) is called the parametric Neumann kernel and is defined by

$$
v(t, s)= \begin{cases}\frac{1}{\pi} \operatorname{Im}\left[\frac{z^{\prime}(t)}{z(t)-z(s)}\right], & t \neq s, \\ \frac{1}{2 \pi} \operatorname{Im}\left[\frac{z^{\prime \prime}(t)}{z^{\prime}(t)}\right], & t=s .\end{cases}
$$

For the proof of existence and uniqueness of the solution of Symm's exterior equation, see [7, pp. 386-388]. But it remains a disadvantage of Symm's exterior equation that the capacity $\gamma$ is unknown. Equation (1.7) shows that $\theta^{\prime}$ is a nonvanishing solution of a certain homogeneous integral equation of the second kind, that is, an eigenfunction. See [7, p. 398] for the proof of existence and uniqueness of the solution of equation (1.8).

See $[\mathbf{1}, \mathbf{8}, \mathbf{1 1}, \mathbf{1 6}]$ for some numerical methods for the computation of the exterior mapping. Excellent surveys on numerical conformal mappings can be found in $[5,7, \mathbf{1 8}]$.

In this paper we present a simple numerical method for computing the boundary values of $\tilde{R}$, and hence $\theta$, in the case when the boundary $\Gamma$ is an analytic Jordan curve. The numerical method is based on a boundary integral equation similar to the Kerzman-Stein integral equation $[\mathbf{1 0}]$ for interior mappings. Typical examples show that numerical results of high accuracy can be obtained. Our method gives $\theta^{\prime}$ and $\gamma$ practically for free.

This paper is organized as follows. In Section 2, we construct an integral equation based on a certain boundary condition satisfied by a function analytic in $\Omega^{-}$. This result is then used in Section 3 to derive an integral equation method for the purpose of exterior mapping. Section 4 gives the numerical implementation, and Section 5 contains examples and numerical results.

2. An integral equation related to a boundary condition. Kerzman and Trummer [10] have derived an integral equation of the second kind, whose unique solution is the Szegö kernel. This integral equation is now called the Kerzman-Stein integral equation by some authors, see $[\mathbf{3}$, p. 108], $[\mathbf{1 7}, \mathbf{1 3}]$, or the integral equation of Kerzman 
and Trummer $[\mathbf{7}$, p. 560]. Perhaps it should be called the KerzmanStein-Trummer integral equation (KST integral equation). Kerzman and Trummer used this integral equation to develop an effective numerical procedure for the construction of the interior mapping function. This procedure may be considered as one of the major advances in the numerical conformal mappings in the past two decades.

Kerzman and Trummer used an operator-theoretic approach (for the derivation of the KST integral equation) that is tantamount to a concrete realization of an operator equation given by Kerzman and Stein [9]. Henrici [7, pp. 560-563] gave a markedly different derivation of the KST integral equation based on a function-theoretic approach. Crucial to Henrici's approach is the use of a certain boundary equation satisfied by the Szegö kernel. Henrici's approach was recently extended by the authors to derive an integral equation satisfied by the Bergman kernel [13]. Motivated by these two cases the authors have also formulated an integral equation related to a general boundary condition satisfied by a function analytic in $\Omega^{+}$such that both the integral equations of the Szegö kernel and the Bergman kernel are special cases.

In this section, we show that a similar general boundary condition satisfied by a function analytic in $\Omega^{-}$can be formulated, on the basis of which an integral equation for the exterior mapping function can be constructed.

Suppose $D(z)$ is analytic with respect to $z \in \Omega^{-}$and is continuous on $\Omega^{-} \cup \Gamma$, where the boundary $\Gamma$ is assumed to be a smooth Jordan curve. We shall assume that $D$ is also Hölder continuous on $\Gamma$. The unit tangent to $\Gamma$ at the point $z(t)$ will be denoted by $T(z)=z^{\prime}(t) /\left|z^{\prime}(t)\right|$. Suppose further that $D$ satisfies the boundary condition

$$
D(z)=c\left[\frac{T(z) Q(z) D(z)}{P(z)}\right]^{-}, \quad z \in \Gamma,
$$

where the minus sign in the superscript denotes complex conjugation, $c$ is a complex constant, $Q$ and $P$ are complex-valued functions with the following properties:

(i) $P(z)$ is analytic with respect to $z \in \Omega^{-}$, is continuous on $\Omega^{-} \cup \Gamma$, and is Hölder continuous on $\Gamma$.

(ii) $P(\infty) \neq 0, D(\infty)$ is finite.

(iii) $P(z) \neq 0, Q(z) \neq 0$, for $z \in \Gamma$. 
Under the above assumptions, we have:

Theorem 1. Let $u$ and $v$ be complex-valued functions defined on $\Gamma$. Then

$$
\begin{aligned}
& \frac{1}{2}\left[v(z)+\frac{u(z)}{\overline{T(z) Q(z)}}\right] D(z) \\
& -\mathrm{PV} \frac{1}{2 \pi i} \int_{\Gamma}\left[\frac{u(z)}{(\bar{w}-\bar{z}) \overline{Q(w)}}-\frac{v(z) T(w)}{w-z}\right] D(w)|d w| \\
& \quad=\operatorname{cu}(z) \frac{\overline{D(\infty)}}{\overline{P(\infty)}}+v(z) D(\infty), \quad z \in \Gamma .
\end{aligned}
$$

Proof. We write

$$
\begin{array}{r}
\mathrm{PV} \frac{1}{2 \pi i} \int_{\Gamma}\left[\frac{u(z)}{(\bar{w}-\bar{z}) \overline{Q(w)}}-\frac{v(z) T(w)}{w-z}\right] D(w)|d w| \\
=\mathrm{PV} \frac{1}{2 \pi i} \int_{\Gamma} \frac{u(z) D(w)}{(\bar{w}-\bar{z}) \overline{Q(w)}}|d w| \\
\quad-\operatorname{PV} \frac{1}{2 \pi i} \int_{\Gamma} \frac{v(z) T(w) D(w)}{w-z}|d w|
\end{array}
$$

and evaluate the two integrals on the right separately. In the second integral, we use $T(w)|d w|=d w$ and the formula [6, p. 27]

$$
\frac{1}{2 \pi i} \int_{\Gamma} \frac{h(w)}{w-z} d w=-\frac{1}{2} h(z)+h(\infty), \quad z \in \Gamma
$$

for a function $h$ which is analytic in $\Omega^{-}$, continuous on $\Omega^{-} \cup \Gamma$, and Hölder continuous on $\Gamma$. Hence we obtain

$$
\mathrm{PV} \frac{1}{2 \pi i} \int_{\Gamma} \frac{v(z) T(w) D(w)}{w-z}|d w|=-\frac{1}{2} v(z) D(z)+v(z) D(\infty)
$$


As for the first integral, we use (2.1), $\overline{T(w)}|d w|=\overline{d w}$ and (2.3) to get

$$
\begin{aligned}
\operatorname{PV} \frac{1}{2 \pi i} \int_{\Gamma} \frac{u(z) D(w)}{(\bar{w}-\bar{z}) \overline{Q(w)}} \mid & |w| \\
& =-c u(z)\left[\operatorname{PV} \frac{1}{2 \pi i} \int_{\Gamma} \frac{D(w)}{(w-z) P(w)} d w\right]^{-} \\
& =-c u(z)\left[-\frac{1}{2} \frac{D(z)}{P(z)}+\frac{D(\infty)}{P(\infty)}\right]^{-} \\
& =\frac{c}{2} u(z) \frac{\overline{D(z)}}{\overline{P(z)}}-c u(z) \frac{\overline{D(\infty)}}{\overline{P(\infty)}} \\
& =\frac{1}{2} u(z) \frac{D(z)}{\overline{T(z) Q(z)}}-c u(z) \frac{\overline{D(\infty)}}{\overline{P(\infty)}}
\end{aligned}
$$

Summarizing,

$$
\begin{aligned}
& \mathrm{PV} \frac{1}{2 \pi i} \int_{\Gamma}\left[\frac{u(z)}{(\bar{w}-\bar{z}) \overline{Q(z)}}-\frac{v(z) T(w)}{w-z}\right] D(w)|d w| \\
&=\frac{1}{2}\left[\frac{u(z)}{\overline{T(z) Q(z)}}+\right.v(z)] D(z) \\
&-c u(z) \frac{\overline{D(\infty)}}{\overline{P(\infty)}}-v(z) D(\infty),
\end{aligned}
$$

which is equivalent to (2.2). This completes the proof.

In what follows it will be convenient to use (2.1) in the equivalent form:

$$
P(z)=\bar{c} \frac{T(z) Q(z) D(z)^{2}}{|D(z)|^{2}}, \quad z \in \Gamma .
$$

In the next section, we apply Theorem 1 to obtain an integral equation related to the exterior mapping. The kernel of this integral equation is the same as the Kerzman-Stein kernel of the KST integral equation.

3. An integral equation method for the exterior mapping. We now assume that the boundary $\Gamma$ of $\Omega^{-}$is an analytic Jordan 
curve. Hence the exterior map $\tilde{R}$ can be extended to a function that is analytic on $\Omega^{-} \cup \Gamma$. Moreover, $\tilde{R}^{\prime}$ is different from zero and analytic in $\Omega^{-} \cup \Gamma \cup\{\infty\}$. Thus a single-valued analytic square root of $\tilde{R}^{\prime}(z)$, denoted by $\sqrt{\tilde{R}^{\prime}(z)}$, may be defined; the square root is chosen such that $\sqrt{\tilde{R}^{\prime}(\infty)}>0[\mathbf{7}$, p. 565]. To justify this, we shall adapt a proof given in $[\mathbf{7}$, p. 375]. We observe that

$$
\log \tilde{R}^{\prime}(z)-\log \tilde{R}^{\prime}\left(z_{0}\right)=\int_{z_{0}}^{z} \frac{\tilde{R}^{\prime \prime}(z)}{\tilde{R}^{\prime}(z)} d z
$$

provided that the above integral defines a single-valued function in $\Omega^{-} \cup \Gamma \cup\{\infty\}$ (since $\Omega^{-}$is not simply connected). As in Henrici [7], we do not replace $z$ in the integrand by a dummy variable; this abuse of notation should not cause any ambiguity. The integral defines a single-valued function provided that

$$
\int_{\Gamma_{1}} \frac{\tilde{R}^{\prime \prime}(z)}{\tilde{R}^{\prime}(z)} d z=0
$$

where $\Gamma_{1}$ is any closed curve surrounding $\Gamma$. In particular, $\Gamma_{1}$ may be taken as a large circle. Since

$$
\frac{\tilde{R}^{\prime \prime}(z)}{\tilde{R}^{\prime}(z)}=O\left(z^{-3}\right), \quad z \rightarrow \infty,
$$

the Laurent series at $\infty$ of $\tilde{R}^{\prime \prime} / \tilde{R}^{\prime}$ has no term in $z^{-1}$, and the integral

$$
\int_{\Gamma_{1}} \frac{\tilde{R}^{\prime \prime}(z)}{\tilde{R}^{\prime}(z)} d z
$$

clearly vanishes. The same expansion shows that by defining

$$
\log \tilde{R}^{\prime}(z):=\log \gamma^{-1}+\int_{\infty}^{z} \frac{\tilde{R}^{\prime \prime}(z)}{\tilde{R}^{\prime}(z)} d z
$$

we obtain an analytic branch of $\log \tilde{R}^{\prime}(z)$ in $\Omega^{-} \cup \Gamma \cup\{\infty\}$. By letting

$$
\sqrt{\tilde{R}^{\prime}(z)}=e^{\left(\log \tilde{R}^{\prime}(z)\right) / 2},
$$


we can also define a single-valued and analytic branch of $\sqrt{\tilde{R}^{\prime}(z)}$.

For $z \in \Gamma$, the exterior map $\tilde{R}(z)$ may be expressed in terms of $\tilde{R}^{\prime}(z)$. To see this, we differentiate both sides of (1.2) with respect to $t$ to obtain

$$
\tilde{R}^{\prime}(z(t)) z^{\prime}(t)=i \theta^{\prime}(t) e^{i \theta(t)} .
$$

Upon division by $\left|\tilde{R}^{\prime}(z(t))\right|\left|z^{\prime}(t)\right|$, we obtain

$$
\tilde{R}(z(t))=-i T(z(t)) \frac{\tilde{R}^{\prime}(z(t))}{\left|\tilde{R}^{\prime}(z(t))\right|},
$$

which is the desired relationship. This relationship also holds if the exterior map $\tilde{R}$ is replaced by the interior map, i.e. the Riemann map [10].

By means of Theorem 1, a corresponding integral equation related to the boundary condition (3.2) may be constructed. The boundary condition (3.2) may be obtained as a special case of (2.4) via the choice of $D(z)=\sqrt{\tilde{R}^{\prime}(z)}, c=i, Q(z)=1$, and $P(z)=\tilde{R}(z)$. From (1.1), $D(\infty)=\sqrt{\tilde{R}^{\prime}(\infty)}=1 / \sqrt{\gamma}$ and $P(\infty)=\tilde{R}(\infty) \neq 0$. Upon substitution into (2.2), along with the choice of $u(z)=\overline{T(z)}$ and $v(z)=1$, we obtain

$$
U(z)-\mathrm{PV} \int_{\Gamma} A(z, w) U(w)|d w|=1, \quad z \in \Gamma,
$$

where

$$
U(z)=\sqrt{\gamma \tilde{R}^{\prime}(z)}
$$

and

$$
A(z, w)=\frac{1}{2 \pi i}\left[\frac{\overline{T(z)}}{\bar{w}-\bar{z}}-\frac{T(w)}{w-z}\right], \quad w, z \in \Gamma, w \neq z .
$$

The kernel $A$ is known as the Kerzman-Stein kernel. It was shown in [10] that for each $z \in \Gamma$, the limit $A(z, w)$ as $w$ approaches $z$ is equal to zero. Hence the Kerzman-Stein kernel is a continuous function 
on $\Gamma \times \Gamma$ if we define $A(z, z)=0$. Thus the symbol PV may be dropped from (3.3). Note that the kernel $A$ is also skew-hermitian, i.e., $A(w, z)=-\overline{A(z, w)}$. Hence, the associated (compact) integral operator has a purely imaginary spectrum. Consequently (3.3) has a unique continuous solution.

As remarked earlier, the kernel $A$ has already served as a kernel of the KST integral equation for the purpose of interior mapping in [10]. The appearance of this kernel once more for the purpose of exterior mapping is rather interesting.

Using (3.2), the boundary values of the exterior map $\tilde{R}$ is evaluated in terms of $U$ by

$$
\tilde{R}(z)=-i T(z) \frac{U(z)^{2}}{|U(z)|^{2}}, \quad z \in \Gamma
$$

Observe that no knowledge of the capacity $\gamma$ is required in (3.6). We now show how the capacity $\gamma$ may be computed from $U$. Using (3.4), we rewrite (3.1) as

$$
\gamma^{-1} U(z(t))^{2} z^{\prime}(t)=i \theta^{\prime}(t) e^{i \theta(t)}
$$

Taking absolute values of both sides yields

$$
\gamma \theta^{\prime}(t)=|U(z(t))|^{2}\left|z^{\prime}(t)\right|
$$

Since

$$
\int_{0}^{\beta} \theta^{\prime}(t) d t=\theta(\beta)-\theta(0)=2 \pi,
$$

integrating both sides of (3.7) with respect to $t$ gives

$$
\gamma=\frac{1}{2 \pi} \int_{0}^{\beta}|U(z(t))|^{2}\left|z^{\prime}(t)\right| d t
$$

If $\theta^{\prime}$ is required, then (3.7) and (3.8) give

$$
\theta^{\prime}(t)=\frac{2 \pi|U(z(t))|^{2}\left|z^{\prime}(t)\right|}{\int_{0}^{\beta}|U(z(t))|^{2}\left|z^{\prime}(t)\right| d t} .
$$


Thus solving (3.3) for $U(z(t))$ yields information about $\theta(t), \theta^{\prime}(t)$, and the capacity $\gamma$.

Another integral equation involving the Neumann kernel related to $\tilde{R}^{\prime}$ may also be obtained. From (3.2) we obtain

$$
\tilde{R}(z)^{2}=-T(z)^{2} \frac{\tilde{R}^{\prime}(z)^{2}}{\left|\tilde{R}^{\prime}(z)\right|^{2}}, \quad z \in \Gamma .
$$

The boundary condition (3.10) may be obtained as a special case of (2.4) via the choice of $D(z)=\tilde{R}^{\prime}(z), c=-1, Q(z)=T(z)$, and $P(z)=\tilde{R}(z)^{2}$. Upon substitution into (2.2), along with the choice of $u(z)=\overline{T(z)^{2}}$ and $v(z)=1$, we obtain

$$
V(z)-\mathrm{PV} \frac{1}{2 \pi i} \int_{\Gamma}\left[\frac{\overline{T(z)^{2}} T(w)}{\bar{w}-\bar{z}}-\frac{T(w)}{w-z}\right] V(w)|d w|=1, \quad z \in \Gamma
$$

where $V(z)=\gamma \tilde{R}^{\prime}(z)$. If we multiply both sides by $T(z)$ and let $W(z)=T(z) V(z)$, we obtain

$$
W(z)-\mathrm{PV} \int_{\Gamma} N(z, w) W(w)|d w|=T(z), \quad z \in \Gamma,
$$

where

$$
N(z, w)= \begin{cases}\frac{1}{\pi} \operatorname{Im}\left[\frac{T(z)}{z-w}\right], & z, w \in \Gamma, z \neq w, \\ \frac{1}{2 \pi} \frac{\operatorname{Im}\left[z^{\prime \prime}(t) \overline{z^{\prime}(t)}\right]}{\left|z^{\prime}(t)\right|^{3}}, & z=w \in \Gamma .\end{cases}
$$

The real kernel $N$ is the familiar Neumann kernel which arises frequently in the integral equations of potential theory and conformal mapping, see, e.g., [7, p. 282]. Since the Neumann kernel $N(w, z)$ is smooth for all $w, z \in \Gamma$, the symbol PV may be dropped from (3.12).

To deal with the question of solvability of the integral equation (3.12), we parametrize $\Gamma$ by $z=z(t), 0 \leq t \leq \beta$. With $w=z(s)$, the integral equation (3.12) will have the form

$$
\phi(t)-\int_{0}^{\beta} v(t, s) \phi(s) d s=\psi(t),
$$


where for $0 \leq s, t \leq 2 \pi$,

$$
\begin{aligned}
\phi(t) & =\left|z^{\prime}(t)\right| W(z(t)), \\
\psi(t) & =z^{\prime}(t),
\end{aligned}
$$

and the kernel $v(t, s)$ is the parametric Neumann kernel (1.9). It is well known, see, e.g., [7, pp. 284-286], that $\lambda=1$ is an eigenvalue of the integral operator whose kernel is $v(t, s)$ with the multiplicity one, and $\phi_{0}(t)=\theta^{\prime}(t)$ is an eigenfunction belonging to this eigenvalue, where $\theta$ is the exterior boundary correspondence function [7, p. 397]. Thus to establish solvability of (3.13), Fredholm theory requires us to consider the adjoint homogeneous equation:

$$
\tilde{\phi}(t)-\int_{0}^{\beta} v(s, t) \tilde{\phi}(s) d s=0 .
$$

It is also known that $\tilde{\phi}(t)=1$ is an eigenfunction of (3.14) and there are no other linearly independent eigenfunctions $[\mathbf{7}$, p. 286]. It thus follows from the Fredholm theory that the inhomogeneous equation (3.13) has a solution if and only if

$$
\int_{0}^{\beta} z^{\prime}(t) \tilde{\phi}(t) d t=\int_{0}^{\beta} z^{\prime}(t) d t=0,
$$

which is indeed true. The Fredholm theory also states that the solution of (3.13) is determined only up to an arbitrary multiple of $\phi_{0}(t)$.

4. Numerical implementation. Using a parametric representation $z(t)$ of $\Gamma, 0 \leq t \leq \beta$, the integral equation (3.3) becomes

$$
\phi(t)-\int_{0}^{\beta} k(t, s) \phi(s) d s=\psi(t),
$$

where for $0 \leq s, t \leq \beta$,

$$
\begin{aligned}
\phi(t) & =\left|z^{\prime}(t)\right|^{1 / 2} U(z(t)), \\
k(t, s) & =\left|z^{\prime}(t)\right|^{1 / 2}\left|z^{\prime}(s)\right|^{1 / 2} A(z(t), z(s)), \\
\psi(t) & =\left|z^{\prime}(t)\right|^{1 / 2} .
\end{aligned}
$$


Observe that the new kernel $k$ preserves the skew-hermitian property.

Since the functions $\phi, \psi$, and $v$ in (4.1) are $\beta$-periodic, an appealing procedure for solving (4.1) numerically is to use the Nyström's method [2] with the trapezoidal rule which is equivalent to the Fourier method [4]. The trapezoidal rule is most accurate for integrating periodic functions [15]. By choosing $n$ equidistant collocation points $t_{i}=$ $(i-1) \beta / n$ and the trapezoidal rule for Nyström's method to discretize (4.1), we obtain

$$
\phi\left(t_{i}\right)-\frac{\beta}{n} \sum_{j=1}^{n} k\left(t_{i}, t_{j}\right) \phi\left(t_{j}\right)=\psi\left(t_{i}\right), \quad 1 \leq i \leq n .
$$

Defining the matrix $B$ by $B_{i j}=\beta k\left(t_{i}, t_{j}\right) / n$ and $x_{i}=\phi\left(t_{i}\right), y_{i}=\psi\left(t_{i}\right)$, equation (4.2) can be rewritten as an $n \times n$ system

$$
(I-B) \mathbf{x}=\mathbf{y} .
$$

Since (4.1) has a unique solution, then for a wide class of quadratures the system (4.3) also has a unique solution, as long as $n$ is sufficiently large [2]. Observe that the complex matrix $B$ is skew-hermitian.

Once the solution $x_{i}=\phi\left(t_{i}\right)$ has been computed, discretization of (4.1) provides us with a very good "natural" interpolation formula [2]:

$$
\phi(t)=\psi(t)+\frac{\beta}{n} \sum_{j=1}^{n} k\left(t, t_{j}\right) \phi\left(t_{j}\right) .
$$

Finally, using (3.6), (3.8), and (3.9), the values of $\tilde{R}(z(t)), \theta(t), \theta^{\prime}(t)$, and the capacity $\gamma$ are computed by the following formulas:

$$
\begin{aligned}
\tilde{R}(z(t)) & =-i T(z(t)) \frac{\phi^{2}(t)}{\left|\phi^{2}(t)\right|}, \\
\theta(t) & =\arg \left(-i \phi^{2}(t) z^{\prime}(t)\right), \\
\theta^{\prime}(t) & =\frac{2 \pi|\phi(t)|^{2}}{\int_{0}^{\beta}|\phi(t)|^{2} d t}, \\
\gamma & =\frac{1}{2 \pi} \int_{0}^{\beta}|\phi(t)|^{2} d t .
\end{aligned}
$$


For boundaries having rotational or axial symmetries, the integral equation (3.3) may be transformed into a new integral equation which depends on a small part of the original boundary. This gives rise to a smaller system of linear equations thereby saving computing time. The details are similar to the case of the interior mapping treated in [19], and will be omitted.

5. Numerical results. In this section the numerical scheme discussed in Section 4 is applied to two test regions that were also considered in $[\mathbf{1}, \mathbf{1 1}, \mathbf{1 6}]$, namely the exteriors of an ellipse and an oval of Cassini. The method employed in solving the system of equations (4.3) is the Gaussian elimination, which has an order of operations of $O\left(n^{3}\right)$ with $n$ being the number of discretization points of the boundary. We do not perform any real conversion when solving the system. The entire computer programming was done using the MATHEMATICA package $[\mathbf{2 0}]$ in single precision (16 digit machine precision).

We list the sup-norm error $\left\|\tilde{R}(z(t))-\tilde{R}_{M}(z(t))\right\|_{\infty}$, where $\tilde{R}(z(t))$ is the exact boundary values and $\tilde{R}_{M}(z(t))$ is the approximation obtained by means of the interpolation formula given in Section 4 using $M$ equally spaced points in the parameter interval, most of which are not the original collocation points. In all our experiments we have chosen $M=36$. Tables 1 and 2 show the numerical results. Tables 3 and 4 compare some of our numerical results with those obtained by the method of Amano [1]. The comparisons show the efficiency of our method for "nearly circular" boundaries.

An efficient iterative procedure for solving a linear system of the form (4.3), where the matrix $B$ is skew-hermitian, is by the conjugate gradient method. This method has an operation count of order $O\left(n^{2}\right)$. For a numerical example of this method in interior mapping, see [19].

Observe that the matrix in the system (4.3) is a full or dense matrix. This type of matrix commonly arises whenever an integral equation is discretized. Various fast algorithms have been developed to answer the challenge of solving linear systems involving full matrices. Fast algorithms should have operation counts of the order not more than $O\left(n \cdot[\log n]^{\mu}\right)$, where $\mu$ is a certain nonnegative constant. Among them is a fast algorithm due to Rokhlin [14] which is based on certain Taylor or Laurent series expansions for the entries of the matrix which are far 
away from the main diagonal. For a recently developed fast algorithm using wavelet approach, see [12].

Example 1. Ellipse $(a \geq 0$, axis ratio $=a)$.

$$
\begin{aligned}
z(t) & =a \cos t+i \sin t, \\
\tilde{R}(z) & =\frac{z+\left(z^{2}-a^{2}+1\right)^{1 / 2}}{a+1} .
\end{aligned}
$$

TABLE 1. Ellipse. Error norm $\left\|\tilde{R}(z(t))-\tilde{R}_{M}(z(t))\right\|_{\infty}$

\begin{tabular}{|rccccccc|}
\hline \multicolumn{7}{|c|}{ axis ratio } \\
\hline$n$ & 1.2 & 1.5 & 2.0 & 3.0 & 5.0 & 10.0 & 20.0 \\
\hline 4 & $6.5(-04)$ & $9.4(-03)$ & $5.9(-02)$ & $2.7(-01)$ & - & - & - \\
8 & $3.6(-07)$ & $3.1(-05)$ & $8.1(-04)$ & $1.7(-02)$ & $1.6(-01)$ & - & - \\
16 & $3.1(-12)$ & $5.7(-09)$ & $8.8(-07)$ & $9.0(-05)$ & $5.7(-03)$ & $1.2(-01)$ & - \\
32 & $7.2(-16)$ & $3.4(-15)$ & $3.5(-11)$ & $5.7(-08)$ & $1.6(-05)$ & $5.9(-03)$ & $1.6(-01)$ \\
64 & - & - & $5.7(-16)$ & $2.8(-13)$ & $5.3(-09)$ & $9.9(-06)$ & $6.4(-03)$ \\
128 & - & - & - & $5.7(-16)$ & $5.2(-15)$ & $3.3(-09)$ & $8.8(-06)$ \\
\hline
\end{tabular}

Example 2. Oval of Cassini $\left(|z-1||z+1|>a^{2}, a>1\right)$.

$$
\begin{aligned}
z(t) & =\left(\cos 2 t+\sqrt{a^{4}-\sin ^{2} 2 t}\right)^{1 / 2} e^{i t}, \\
\tilde{R}(z) & =\frac{\left(z^{2}-1\right)^{1 / 2}}{a} .
\end{aligned}
$$

TABLE 2. Oval of Cassini. Error norm $\left\|\tilde{R}(z(t))-\tilde{R}_{M}(z(t))\right\|_{\infty}$

\begin{tabular}{|rcccccc|}
\hline \multicolumn{7}{c|}{ values of $a$} \\
\hline $\mathrm{n}$ & 5 & 2 & 1.25 & 1.11 & 1.0101 & 1.001001 \\
\hline 4 & $9.9(-06)$ & $2.5(-03)$ & $5.1(-02)$ & - & - & - \\
8 & $3.1(-09)$ & $3.2(-05)$ & $5.9(-03)$ & $3.4(-02)$ & - & - \\
16 & $1.4(-15)$ & $6.3(-09)$ & $7.6(-05)$ & $1.7(-03)$ & - & - \\
32 & - & $2.5(-14)$ & $1.6(-08)$ & $5.6(-06)$ & $1.7(-02)$ & - \\
64 & - & $4.0(-16)$ & $1.8(-15)$ & $8.7(-11)$ & $4.9(-04)$ & - \\
128 & - & - & - & $7.0(-16)$ & $5.6(-07)$ & $4.2(-03)$ \\
\hline
\end{tabular}


TABLE 3. Ellipse. Error norm $\|\cdot\|_{\infty}$

\begin{tabular}{|rcc|rcc|}
\hline$a$ & $n$ & our method & $\mathrm{a}$ & $\mathrm{n}$ & Amano's method \\
\hline 1.25 & 32 & $1.2(-15)$ & 1.25 & 64 & $7.4(-13)$ \\
2.5 & 64 & $1.9(-15)$ & 2.5 & 64 & $3.1(-07)$ \\
5 & 64 & $5.3(-09)$ & 5 & 64 & $5.4(-05)$ \\
10 & 64 & $9.9(-06)$ & 10 & 64 & $1.3(-03)$ \\
20 & 64 & $6.4(-03)$ & 20 & 64 & $6.9(-03)$ \\
\hline
\end{tabular}

TABLE 4. Oval of Cassini. Error norm $\|\cdot\|_{\infty}$

\begin{tabular}{|rcc|rcc|}
\hline$a$ & $\mathrm{n}$ & our method & $a$ & $n$ & Amano's method \\
\hline 1.8 & 32 & $1.3(-14)$ & 1.8 & 64 & $2.4(-13)$ \\
1.06 & 64 & $3.3(-08)$ & 1.06 & 64 & $2.1(-08)$ \\
1.00995 & 64 & $5.1(-04)$ & 1.00995 & 64 & $9.1(-05)$ \\
\hline
\end{tabular}

\section{REFERENCES}

1. K. Amano, A charge simulation method for the numerical conformal mapping of interior, exterior and doubly-connected domains, J. Comp. Appl. Math. 53 (1994), 353-370.

2. K.E. Atkinson, A survey of numerical methods for the solution of Fredholm integral equations, Society for Industrial and Applied Mathematics, Philadelphia, 1976.

3. S.R. Bell, The Cauchy transform, potential theory, and conformal mapping, CRC Press, Boca Raton, Florida, 1992.

4. J.P. Berrut and M. R. Trummer, Equivalence of Nyström's method and Fourier methods for the numerical solution of Fredholm integral equations, Math. Comp. 48 (1987), 617-623.

5. D. Gaier, Konstruktive Methoden der Konformen Abbildung, Springer, Heidelberg, 1964.

6. F.D. Gakhov, Boundary value problems, English translation of Russian edition 1963 (I.N. Sneddon, ed.), Pergamon Press, New York, 1966.

7. P. Henrici, Applied and computational complex analysis, Vol 3., John Wiley, New York, 1986.

8. D.M. Hough and N. Papamichael, An integral equation method for the numerical conformal mapping of interior, exterior and doubly-connected domains, Numer. Math. 41 (1983), 287-307.

9. N. Kerzman and E. M. Stein, The Cauchy kernel, the Szegö kernel, and the Riemann mapping function, Math. Ann. 236 (1978), 85-93. 
10. N. Kerzman and M. R. Trummer, Numerical conformal mapping via the Szegö kernel, J. Comp. Appl. Math. 14 (1986), 111-123.

11. P. Rabinowitz, Numerical experiments in conformal mapping by the method of orthonormal polynomials, J. Assoc. Comp. Mach. 13 (1966), 296-303.

12. A. Rathsfeld, A wavelet algorithm for the solution of the double layer potential equation over polygonal boundaries, J. Integral Equations Appl. 7 (1995), 47-98.

13. M.R.M. Razali, M.Z. Nashed and A.H.M. Murid, Numerical conformal mapping via the Bergman kernel, J. Comp. Appl. Math. 82 (1997), 333-350.

14. V. Rokhlin, Rapid solution of integral equations of classical potential theory, J. Comput. Phys. 60 (1985), 187-207.

15. E. Stiefel, On solving Fredholm integral equations, SIAM J. Appl. Math. 4 (1955), 63-85.

16. G.T. Symm, Numerical mapping of exterior domains, Numer. Math. 10 (1967), 437-445.

17. A.D. Thomas, Conformal mapping of nonsmooth domains via the KerzmanStein integral equation, J. Math. Anal. Appl. 200 (1996), 162-181.

18. L.N. Trefethen, ed., Numerical conformal mapping, North-Holland, Amsterdam, 1996

19. M.R. Trummer, An efficient implementation of a conformal mapping method based on the Szegö kernel, SIAM J. Numer. Anal. 23 (1986), 853-872.

20. S. Wolfram, Mathematica: A system of doing mathematics by computer, Addison-Wesley, Redwood City, 1991.

Department of Mathematics, Faculty of Science, Universiti Teknologi Malaysia, KB 791, 80990 Johor Bahru, Malaysia.

E-mail address: alihmm@math.fs.utm.my

Department of Mathematical Sciences, University of Delaware, Newark, DelaWARE 19716, USA.

E-mail address: nashed@math.udel.edu

Department of Mathematics, Faculty of Science, Universiti Teknologi Malaysia, KB 791, 80990 Johor Bahru, Malaysia.

E-mail address: s-rshdi@fs.utm.my 